\title{
Evaluation of Dimensional Accuracy of Three Combinations of Polyvinyl Siloxane Impression Material: An In Vitro Study
}

Preetam A Mahagaonkar ${ }^{1}$, Prabhakar B Angadi², Nihar R Naik ${ }^{3}$, Vinay Kakatkar ${ }^{4}$, Sushilamma H Manjunath ${ }^{5}$, Yogesh S Sonawane ${ }^{6}$

\begin{abstract}
Aim: The aim of the study was to evaluate the dimensional accuracy of three combinations of polyvinyl siloxane impression material by doublemix single-step impression technique.

Materials and methods: Metal master model was made according to the ADA specification no. 19; ISO 4823:2000/AMD 2007. Impressions were made using perforated custom-made metallic trays of $2 \mathrm{~mm}$ and $4 \mathrm{~mm}$ spacing, the impression materials used were putty, heavy body, regular body and light body. A total of 30 impressions were made by single-step technique and poured in die stone to obtain resultant cast. Ten impressions were made of each combination of polyvinyl siloxane (PVS). Three dimensions (interabutment distance, height and diameter) on resultant cast were measured and compared with metal master model. The results were statistically analyzed and tabulated.

Results: Diameter of abutment, the height of abutment and interabutment distance in each group were larger in dimensions as compared with metal master model. The dimensional discrepancies of group I, group II and group III casts when compared with the master model were significantly different from each other. The least difference was found in group I.

Conclusion: The one-step putty-light body combination (group I) produced the most accurate stone casts compared with one-step heavy body-light body and regular body-light body combinations.

Clinical significance: In everyday dental practice, impression making is imperative. Hence, by doing this study, we tried to find out which material combination is suitable to give us predictable and accurate results.

Keywords: Dimensional accuracy, Double-mix single-step technique, Polyvinyl siloxane impression material, Viscosity.

The Journal of Contemporary Dental Practice (2020): 10.5005/jp-journals-10024-2746
\end{abstract}

\section{INTRODUCTION}

Impression making to duplicate oral condition and tooth morphology is an integral part of prosthetic dentistry. Both, material and technique are important elements of impression accuracy. ${ }^{1}$

Production of excellent crowns and fixed partial dentures depends upon the usage of impression material and its technique, that accurately reproduces prepared teeth and their relationship to the adjacent hard and soft tissues of the oral cavity. ${ }^{2}$

A variety of dental impression materials are available such as alginate, agar and elastomers.

Four basic types of elastomeric impression materials are currently in use: polysulphides, polyether, condensation silicones and addition silicones. ${ }^{3}$

The ADA specification no. 19; ISO4823:2000/AMD2007 classified elastomeric impression materials in: type I, II, or III according to the properties after setting. ${ }^{4}$ These properties are elastic recovery of the material after a compressive strain, the resistance to distortion under a small compressive stress and the total linear dimensional change of a specimen after a minimum of 24 hours in unstressed condition.

Out of different elastomers, polyvinyl siloxane or addition silicones have demonstrated superior physical properties and have attained clinical success. Improvements in accuracy and dimensional stability, surface quality, elastic recovery, flow flexibility, hydrophilicity and tear strength have contributed to the continued increasing use and success of vinyl polysiloxane material. ${ }^{5}$

Different clinical situations demand the utilization of different impression materials and techniques, with each one having its own merits and demerits. This study was undertaken to understand and analyze the dimensional accuracy of the three combinations of vinyl
1,2Department of Prosthodontics, SMBT Dental College and Hospital, Sangamner, Maharashtra, India

${ }^{3}$ Department of Prosthodontics, Chhattisgarh Dental College and Research Institute, Rajnandgaon, Chhattisgarh, India

${ }^{4}$ Department of Prosthodontics, SMBT Institute of Dental Sciences and Research, Nashik, Maharashtra, India

${ }^{5}$ Department of Periodontology, SMBT Dental College and Hospital, Sangamner, Maharashtra, India

${ }^{6}$ Department of Prosthodontics, Sub District Hospital, Karjat, Ahmednagar, Maharashtra, India

Corresponding Author: Preetam A Mahagaonkar, Department of Prosthodontics, SMBT Dental College and Hospital, Sangamner, Maharashtra, India, Phone: +91 9960535822, e-mail: preetam.am@ gmail.com

How to cite this article: Mahagaonkar PA, Angadi PB, Naik NR, et al. Evaluation of Dimensional Accuracy of Three Combinations of Polyvinyl Siloxane Impression Material: An In Vitro Study. J Contemp Dent Pract 2020;21(2):190-196.

Source of support: Nil

Conflict of interest: None

polysiloxane impression materials (i.e., putty + light body, heavy body + light body and regular body + light body) by double-mix single-step impression technique.

\section{Materials and Methods}

This study was conducted at Rural Dental College, Loni, Ahmednagar, Maharashtra.

() The Author(s). 2020 Open Access This article is distributed under the terms of the Creative Commons Attribution 4.0 International License (https://creativecommons. org/licenses/by-nc/4.0/), which permits unrestricted use, distribution, and non-commercial reproduction in any medium, provided you give appropriate credit to the original author(s) and the source, provide a link to the Creative Commons license, and indicate if changes were made. The Creative Commons Public Domain Dedication waiver (http://creativecommons.org/publicdomain/zero/1.0/) applies to the data made available in this article, unless otherwise stated. 
A metal model with two tapered abutment preparation having a base of $5 \mathrm{~mm}$ was prepared using a lathe machine. The abutments were designated as " $X$ " and " $Y$ ". The abutments were prepared with cross-groves on occlusal surface for reference measurements. The points obtained by intersection of two lines at $90^{\circ}$ to each other was named as point " $X$ " for abutment $X$ and point " $Y$ " for abutment Y. The abutments were attached to the metal base of the model which was $5 \mathrm{~mm}$ in thickness. This was then used as definitive standardized model for the impression making in the study (Fig. 1).

Two custom made metallic perforated trays were fabricated, one for impression making of putty-light body combination with multiple holes of diameter $2 \mathrm{~mm}$ and the inner walls of the tray was at a distance of $4 \mathrm{~mm}$ from the abutments. The other custom tray was fabricated for impression making of heavy body-light body combination, as well as for regular body-light body combination. The tray was prepared with multiple holes of diameter $2 \mathrm{~mm}$ and the inner walls of the tray were at a distance of $2 \mathrm{~mm}$ from the abutments (Fig. 2).

A total of 30 impressions were made and poured in die stone to obtain resultant cast. All the impressions were made using impression material of Reprosil, Dentsply Caulk company.

\section{Making of Impression}

For each combination of materials, 10 impressions of the metal master model were made by double-mix single-step impression technique (one step). Impressions were inspected for the flow of the material, presence of any voids and was poured in improved die stone using water powder ratio recommended by the manufacturer.

The impression making steps of various study groups were as follows:

\section{Group "I": Putty-Light Body Combination}

The metal master model was cleaned with gauge piece and tray adhesive was applied to the perforated metallic tray evenly and was allowed to dry for 10 minutes. The putty was kneaded and loaded onto the tray, at the same time the light body was manipulated and dispensed onto the abutments of metal master model. Now the tray with putty was seated onto the abutments with proper orientation. The finger pressure was applied to make the tray border and the metal model to come in metal to metal contact. The entire assembly was clamped to prevent any further displacement of the tray. The material was allowed to set for 12 minutes, then the impression was removed and examined (Fig. 3).

\section{Group "II": Heavy Body-Light Body Combination}

Prior preparation was same as that of putty-light body impression procedure. Here heavy body material was utilized as tray material and the light body material was utilized as the syringe material. The heavy body was mixed on the glass slab with the help of spatula and was loaded onto the tray, at the same time the

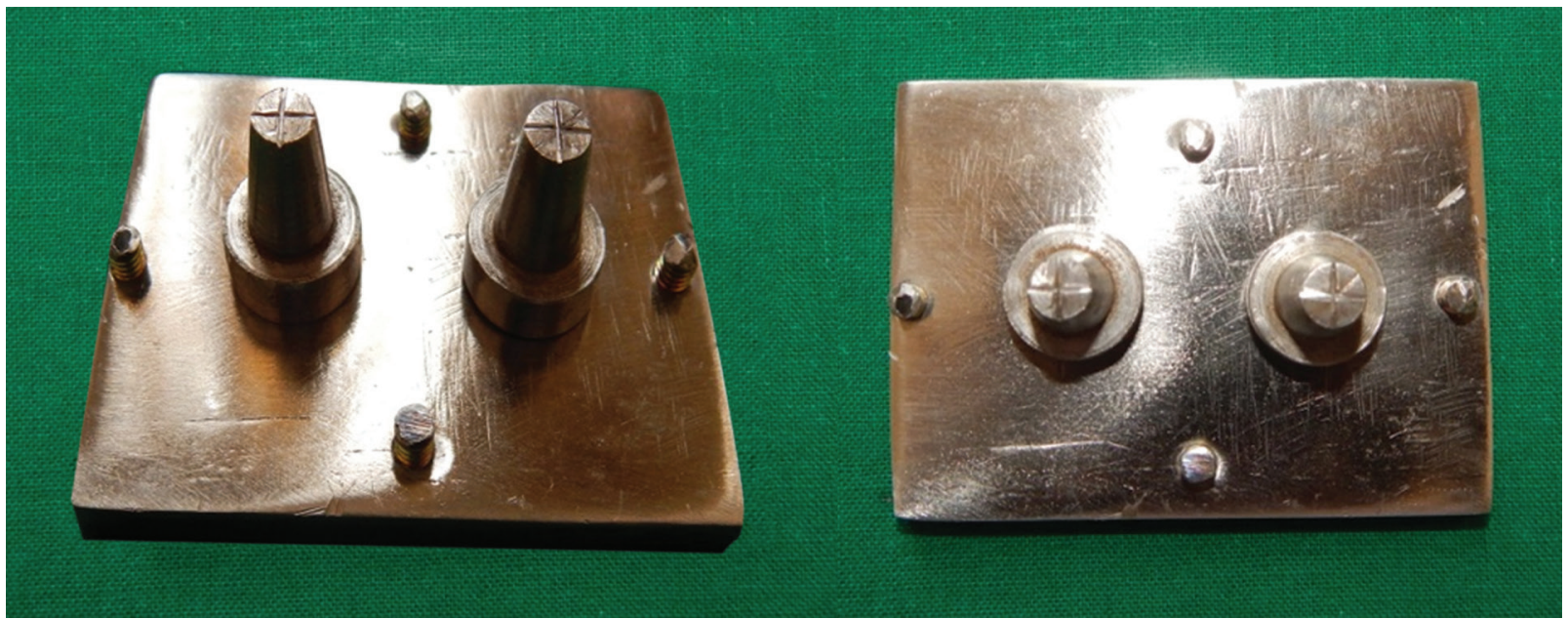

Fig. 1: Metal master model
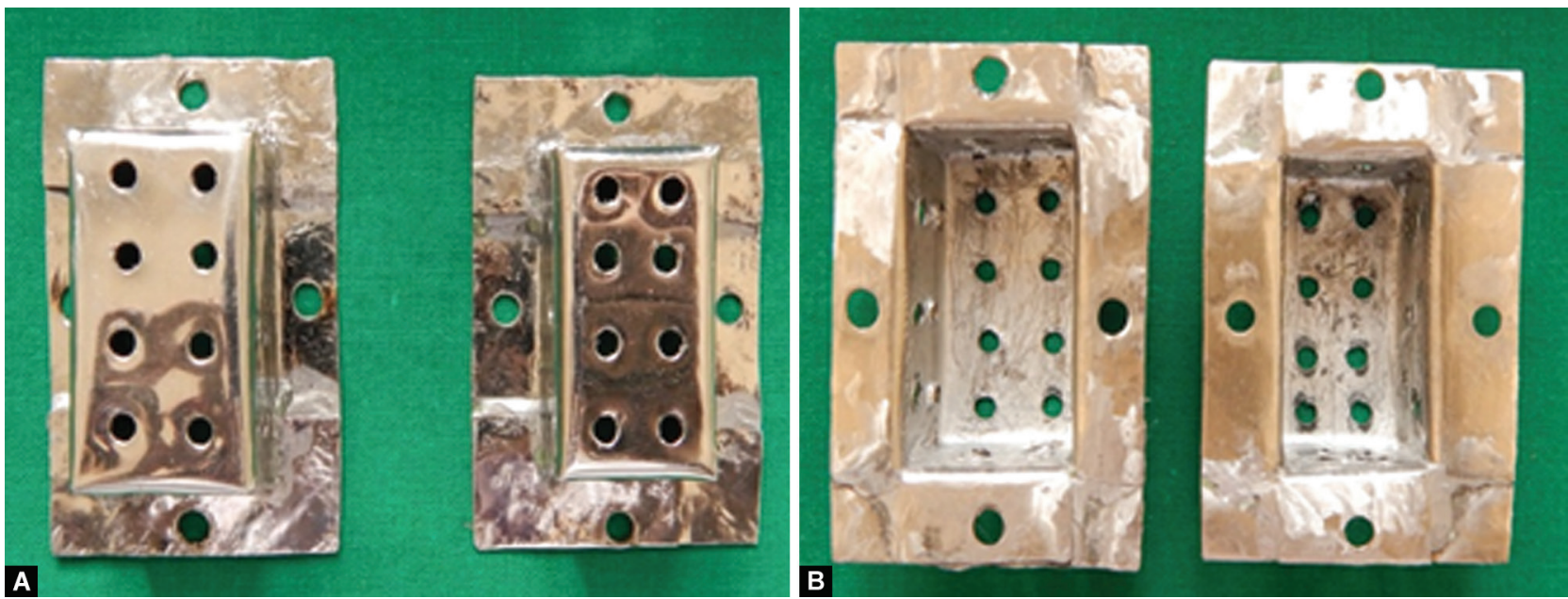

Figs $2 \mathrm{~A}$ and B: (A) Perforated metallic custom tray, $4 \mathrm{~mm}$ spacing and $2 \mathrm{~mm}$ spacing; (B) Inverted view 


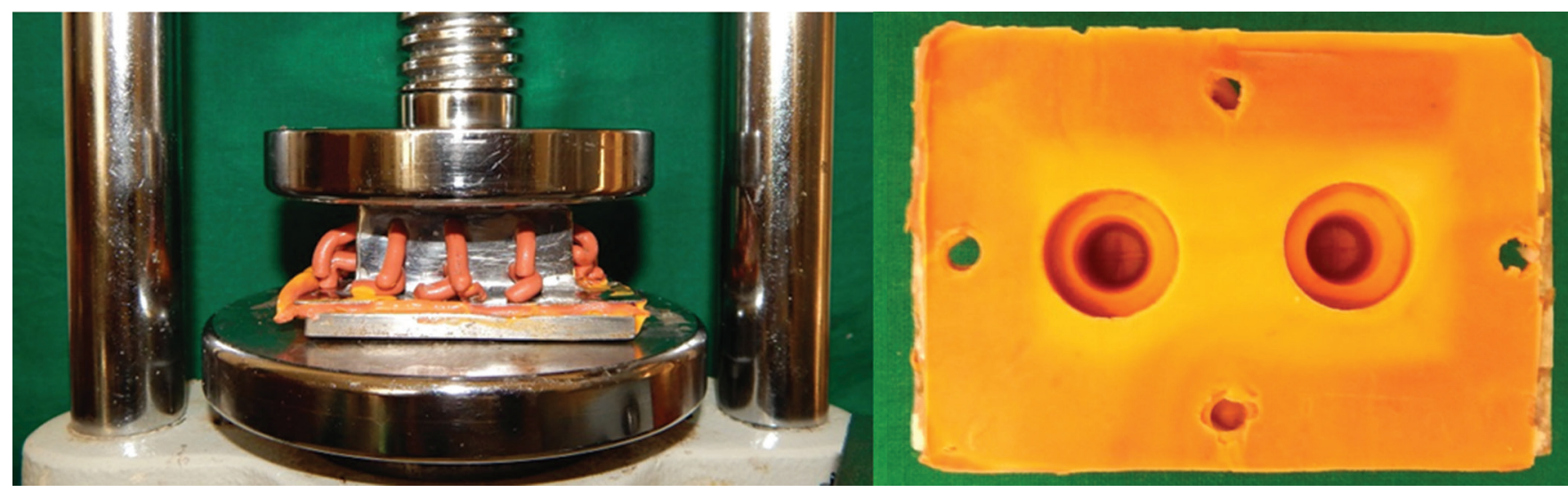

Fig. 3: Putty-light body combination

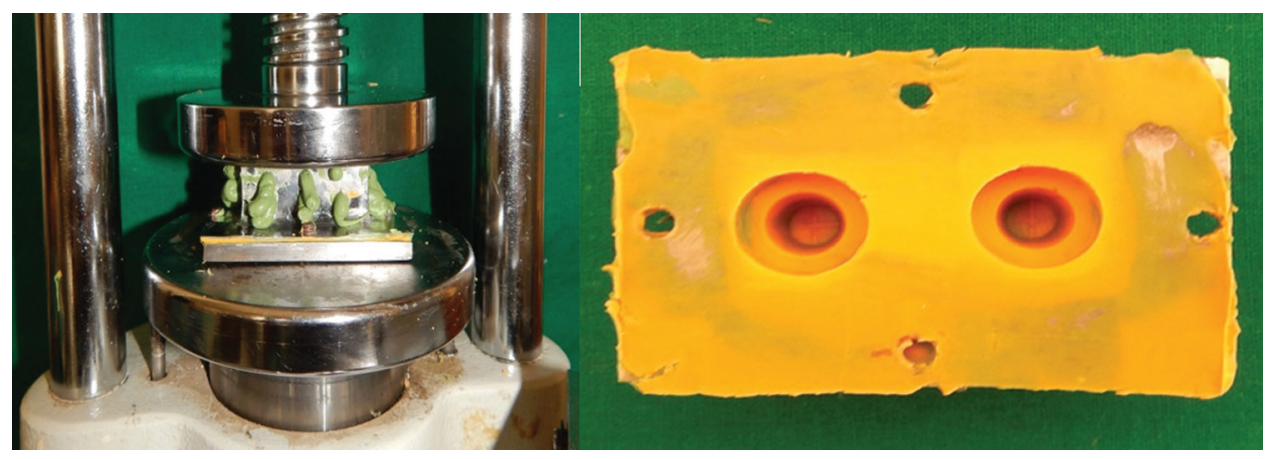

Fig. 4: Heavy body-light body combination

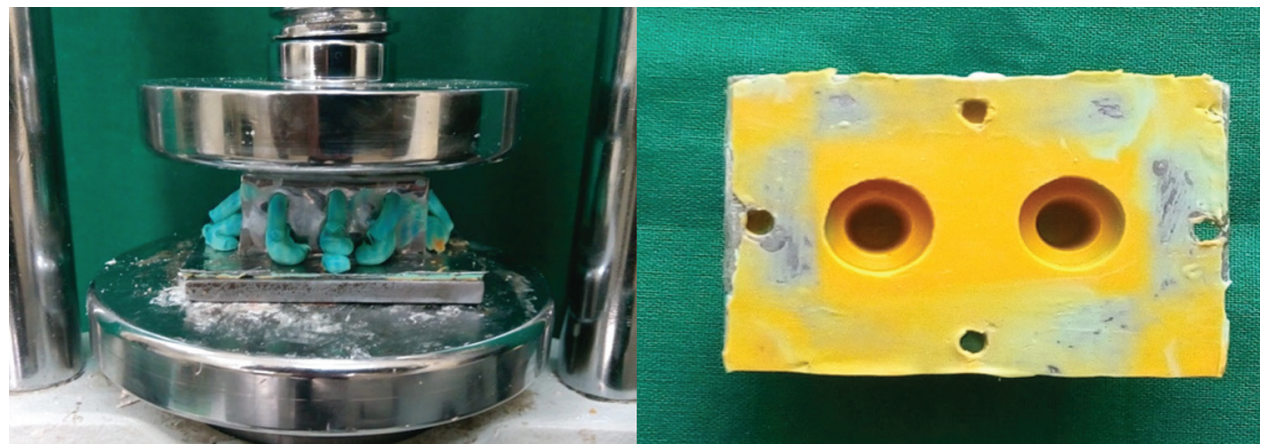

Fig. 5: Regular body-light body combination

light body was manipulated and dispensed into the abutments of metal model. Rest of the procedure was the same as that in group I (Fig. 4).

\section{Group "III": Regular Body-Light Body Combination}

Prior preparation was same as that of putty-light body impression procedure. Here regular body material was utilized as tray material and the light body material was utilized as the syringe material. The regular body was mixed on the glass slab with the help of spatula and was loaded onto the tray, at the same time the light body was manipulated and dispensed onto the abutments of metal model. Rest of the procedure was the same as that in group I (Fig. 5).

All the impressions were made according to the manufacturer's instructions at room temperature. The setting time prescribed by the manufacturer was doubled to compensate for impression making at room temperature instead of at mouth temperature as setting time might differ outside mouth.

All impressions were stored at room temperature for one hour before pouring in the improved stone type IV (Die Stone, Kalrock Kalabhai Karson Pvt. Ltd) for addition silicone.

Firstly, boxing of impression was done, then a ratio of $25 \mathrm{~mL}$ water and $100 \mathrm{~g}$ of improved stone powder was used. The improved stone was initially mixed by hand to incorporate the water and was then mechanically mixed under vacuum for 15 seconds with the help of vacuum mixing unit.

All the mixes were vibrated into the impression, and the stone models were allowed to set for 1 hour before they were separated from the impressions. All the stone models were trimmed mechanically with a model trimmer to an equal thickness and numbered. 
The mean dimensions of metal master model were considered as control group. The impressions made by putty-light body combination were considered as group I, the heavy body-light body combination impressions were considered as group II, and the regular body-light body combination impressions were considered as group III.

A total of 30 casts were used for the study. Ten impressions of each combination of materials (group) were poured and casts were obtained. The accuracy of the impression material was assessed by measuring three dimensions on the stone cast obtained from impressions of the metal master model. The measurements were made by using travelling microscope with a least count of $0.001 \mathrm{~mm}$. The location of the distance measured were the interabutment distance from the center of the abutment $X$ to the center of abutment $Y$ and was designated as $X-Y$, the height of the abutment, the diameter of the abutment (Fig. 6).

\section{Results}

The data was coded and entered into Microsoft Excel spreadsheet. Analysis was done using SPSS version 15 (SPSS Inc. Chicago, IL, USA) Windows software program. The variables were assessed for normality using the Kolmogorov-Smirnov test. Descriptive statistics included computation of means and standard deviations. The independent " $t$ " test and analysis of variance (ANOVA) were used for comparison of all clinical indicators. Analysis of variance (ANOVA) was used to compare the means obtained when the number of groups is more than two. Independent " $t$ " test was used when the number of groups is two. Level of significance was set at $p=0.05$. ( $p=<0.05$, significant; $p=<0.01$, highly significant; $p=<0.001$, very highly significant).

Table 1, shows dimensions of metal master model which was used as a control group in this study.

In Table 2, the results showed that for group I (putty-light body) and group II (heavy body-light body), when the mean interabutment distance was compared with the metal master die it was statistically insignificant. Whereas the results showed that for group III (regular body-light body), the $p$ value was $<0.001$ which indicates that it was highly significant (Fig. 7).

In Table 3, when mean interabutment distance of group I and group II were compared among each other, the $p$ value obtained was 0.31 which indicates that it was insignificant. Whereas mean interabutment distance of group I and group II when compared with group III, respectively the $p$ value obtained was $<0.001$ which indicates that it was significant. Thus, it can be said that group I and group II are almost equally accurate and group III varied in accuracy statistically.

In Table 4, the results showed that for group I (putty-light body), II (heavy body-light body) and group III (regular body-light body), when the mean height was compared with the metal master die it was highly significant (Fig. 8).

In Table 5, when mean height of group I was compared with group II and group III, the $p$ value obtained was 0.48 and 0.27 respectively which indicates that it was insignificant. But when mean height of group II and group III were compared among each other, the $p$ value obtained was 0.05 , which indicates that it is significant. The values of height obtained in group II and group III dies, did show a significant difference between them. Thus, it can be said that group II and group III varied in accuracy statistically.

In Table 6, the results showed that for group I (putty-light body), II (heavy body-light body) and group III (regular body-light body), when the mean diameter was compared with the metal master die, the $p$ value was $<0.001$ which indicates that it is highly significant (Fig. 9).

In Table 7, when mean diameter of group I and group II were compared among each other, the $p$ value obtained was 0.92 which indicates that it is insignificant. Thus, it can be said that group I and group II are almost equally accurate statistically. But when mean diameter of group I and group III and also group II and group III were compared among each other, the $p$ value obtained was $<0.001$ which indicates that it is significant. Thus, it can be said that group I and group III and also group II and group III varied in accuracy statistically amongst each other. This study showed that for all groups, larger dimensions were observed when compared with the metal master model.

Table 1: Dimensions of metal master die (control group)

\begin{tabular}{lll}
\hline $\begin{array}{l}\text { Interabutment } \\
\text { distance }(X-Y)\end{array}$ & Height of abutment & Diameter of abutment \\
\hline $26.07 \mathrm{~mm}$ & $11.50 \mathrm{~mm}$ & $6.50 \mathrm{~mm}$ \\
\hline
\end{tabular}
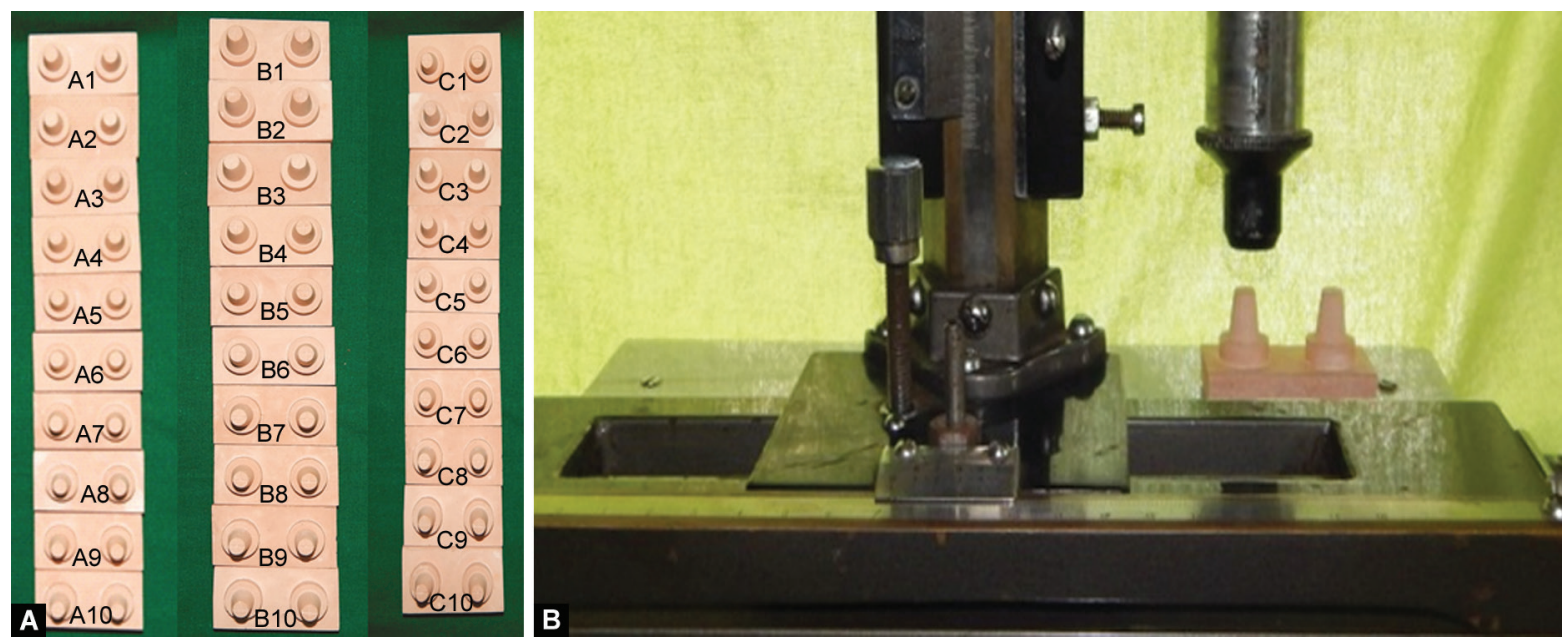

Figs 6A and B: (A) Group I, II and III stone die casts; (B) Traveling microscope with stone die cast 
Table 2: Comparison of interabutment distance (control vs group I, II and III)

\begin{tabular}{lllllc}
\hline \multirow{2}{*}{ Group } & & \multicolumn{3}{l}{ Std. } & Mean \\
Control $26.07 \mathrm{~mm}$ & A & 26.08 & 0.06 & 0.011 & 0.19 \\
& B & 26.10 & 0.046 & 0.360 & 0.38 \\
& C & 26.15 & 0.036 & 0.088 & $<0.001^{*}$ \\
\hline
\end{tabular}

Test applied: ANOVA. *Significant

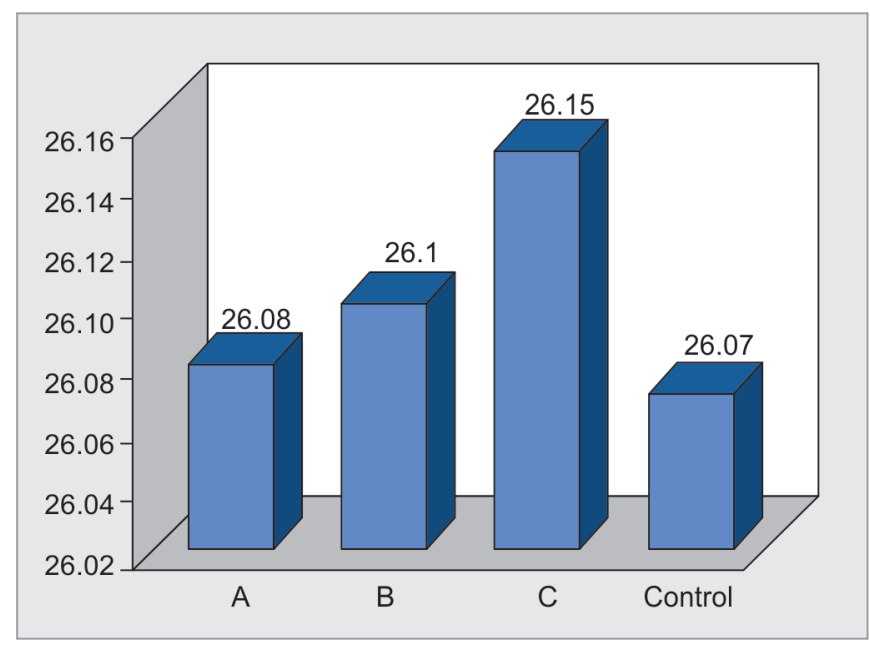

Fig. 7: Comparison of interabutment distance

Table 3: Comparison of interabutment distance: group I, group II and group III

\begin{tabular}{|c|c|c|c|c|c|}
\hline Group & $n$ & Mean $(\mathrm{mm})$ & $\begin{array}{l}\text { Std. } \\
\text { deviation }\end{array}$ & $\begin{array}{l}\text { Mean } \\
\text { difference }\end{array}$ & $p$ value \\
\hline \multicolumn{6}{|c|}{ Group I vs group II } \\
\hline I & 10 & 26.08 & 0.06 & 0.25 & 0.31 \\
\hline II & 10 & 26.10 & 0.04 & & \\
\hline \multicolumn{6}{|c|}{ Group I vs group III } \\
\hline A & 10 & 26.08 & 0.06 & 0.077 & $<0.001^{*}$ \\
\hline C & 10 & 26.15 & 0.03 & & \\
\hline \multicolumn{6}{|c|}{ Group II vs group III } \\
\hline B & 10 & 26.10 & 0.046 & 0.052 & $<0.01^{*}$ \\
\hline C & 10 & 26.15 & 0.036 & & \\
\hline
\end{tabular}

Test applied: independent " $t$ " test. *Significant

Table 4: Comparison of height (control vs group I, II and III)

\begin{tabular}{llllll}
\hline \multirow{2}{*}{ Group } & & \multicolumn{3}{c}{ Std. } & Mean \\
Control 11.5 mm & A & 11.61 & 0.06 & 0.11 & $<0.001^{*}$ \\
& B & 11.59 & 0.05 & 0.09 & $<0.001^{*}$ \\
& C & 11.64 & 0.03 & 0.14 & $<0.001^{*}$ \\
\hline
\end{tabular}

Test applied: ANOVA. *Significant

\section{Discussion}

Dimensional accuracy and stability of impressions are very important for precise fitting of prostheses. Many of the restorative devices used in dentistry are fabricated outside the mouth on the cast i.e., positive replica. ${ }^{4}$ It is neither possible nor desirable to make patterns for fixed prostheses directly in the mouth. ${ }^{6}$

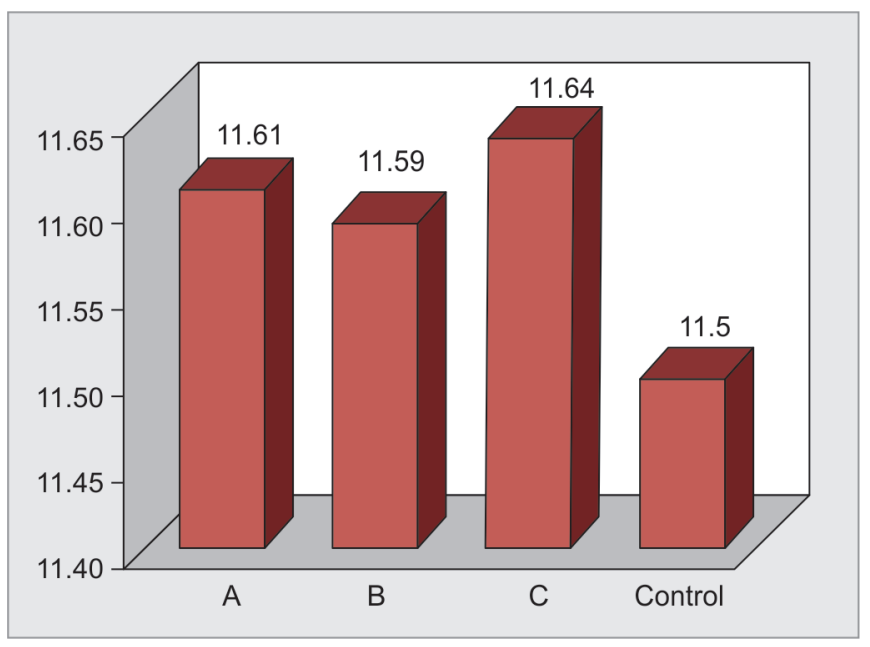

Fig. 8: Comparison of height

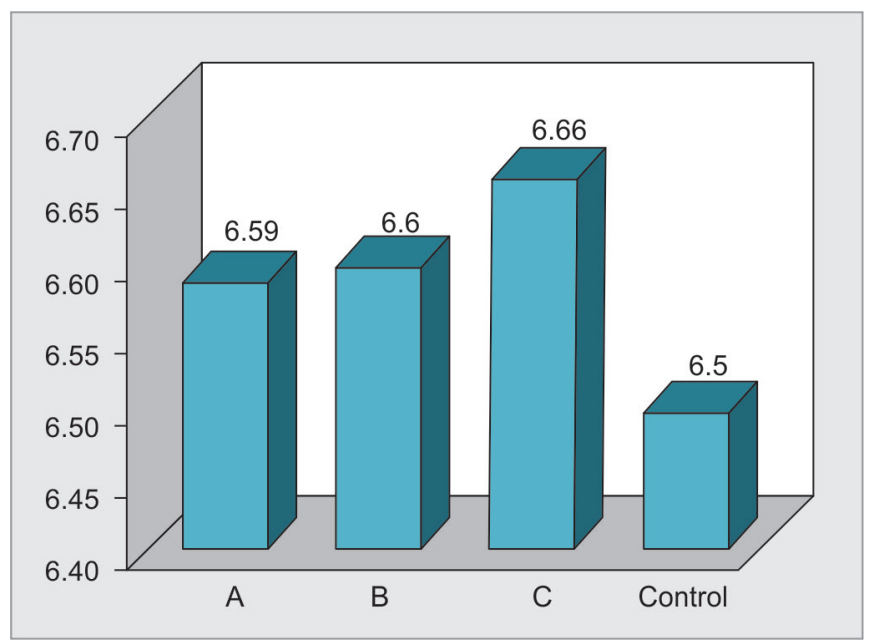

Fig. 9: Comparison of diameter

Table 5: Comparison of height: group I, group II and group III

\begin{tabular}{|c|c|c|c|c|c|}
\hline Group & $n$ & Mean $(\mathrm{mm})$ & $\begin{array}{l}\text { Std. } \\
\text { deviation }\end{array}$ & $\begin{array}{l}\text { Mean } \\
\text { difference }\end{array}$ & $p$ value \\
\hline \multicolumn{6}{|c|}{ Group I vs group II } \\
\hline A & 10 & 11.61 & 0.06 & 0.019 & 0.48 \\
\hline B & 10 & 11.59 & 0.05 & & \\
\hline \multicolumn{6}{|c|}{ Group I vs group III } \\
\hline A & 10 & 11.61 & 0.06 & 0.26 & 0.27 \\
\hline C & 10 & 11.64 & 0.03 & & \\
\hline \multicolumn{6}{|c|}{ Group II vs group III } \\
\hline B & 10 & 11.59 & 0.05 & 0.45 & $0.05^{*}$ \\
\hline C & 10 & 11.64 & 0.03 & & \\
\hline
\end{tabular}

Test applied: independent " $t$ " test. * Significant

Table 6: Comparison of diameter (control vs group I, II and III)

\begin{tabular}{|c|c|c|c|c|c|}
\hline Group & $n$ & Mean $(\mathrm{mm})$ & $\begin{array}{l}\text { Std. } \\
\text { deviation }\end{array}$ & $\begin{array}{l}\text { Mean } \\
\text { difference }\end{array}$ & $p$ value \\
\hline \multirow[t]{3}{*}{ Control $6.5 \mathrm{~mm}$} & A & 6.59 & 0.047 & 0.099 & $<0.001^{*}$ \\
\hline & B & 6.60 & 0.048 & 0.101 & $<0.001^{*}$ \\
\hline & C & 6.66 & 0.041 & 0.164 & $<0.001^{*}$ \\
\hline
\end{tabular}

Test applied: ANOVA. *Significant 
Table 7: Comparison of diameter: group I, group II and group III

\begin{tabular}{|c|c|c|c|c|c|}
\hline Group & $n$ & Mean $(\mathrm{mm})$ & $\begin{array}{l}\text { Std. } \\
\text { deviation }\end{array}$ & $\begin{array}{l}\text { Mean } \\
\text { difference }\end{array}$ & $p$ value \\
\hline \multicolumn{6}{|c|}{ Group I vs group II } \\
\hline A & 10 & 6.599 & 0.047 & 0.002 & 0.92 \\
\hline B & 10 & 6.601 & 0.048 & & \\
\hline \multicolumn{6}{|c|}{ Group I vs Group III } \\
\hline A & 10 & 6.59 & 0.047 & 0.065 & $<0.001^{*}$ \\
\hline C & 10 & 6.66 & 0.041 & & \\
\hline \multicolumn{6}{|c|}{ Group II vs Group III } \\
\hline B & 10 & $6.60 \mathrm{~mm}$ & 0.048 & 0.063 & $<0.001^{*}$ \\
\hline C & 10 & $6.66 \mathrm{~mm}$ & 0.041 & & \\
\hline
\end{tabular}

Test applied: independent ' $t$ ' test. *Significant

Production of exemplary crowns depends upon the usage of impression material and its technique of usage that accurately reproduces the prepared teeth and their relationship to the adjacent oral structure.

PVS impression materials are extremely popular because of their combination of excellent physical properties, handling characteristics and dimensional stability. The addition type silicone impression material PVS has been reported to be most accurate and dimensionally stable. PVS elastomeric impression materials can be used either by one step or two step techniques.

The aim of this study was focused on the effect of three tray material viscosities in combination with light body syringe material of PVS on dimensional accuracy. The accuracy of casts produced by these combinations was compared and efforts were made to standardize most of the variables.

In this study, the dies made by putty-light body combination were considered as group I, the dies made by heavy body-light body combination were considered as group II and the dies made by regular body-light body combination were considered as group III. Heavy body-light body combination and regular body-light body combination impressions were made with the help of a custom tray of $2 \mathrm{~mm}$ spacing whereas putty-light body combination impression was made with custom tray of $4 \mathrm{~mm}$ spacing.

The interabutment distance, diameter and height of the metal master model, i.e., control group, group I, group II and group III were measured.

To reduce the number of factors that could have influenced the outcome, impression materials were used of the same company i.e., Reprosil from Dentsply Caulk for all the groups.

In present study all the impressions were made by one step technique, as it is less time consuming, saves material and is simple to perform. According to study conducted by Hung et al. ${ }^{8}$ accuracy of addition silicone impression material is affected more by the material than technique and also accuracy of putty wash one step technique was not different from putty wash two step technique. The difference between single and double mix in accuracy and elasticity may be attributed to the rate of polymerization: faster polymerization will prohibit the penetration of free radicals. The light body component occupies minute volume, so its effect on the resultant deformation will be slight. ${ }^{21}$

The impressions were made using metal custom trays of $2 \mathrm{~mm}$ and $4 \mathrm{~mm}$ spacing as, Balkenhol et al. ${ }^{12}$ concluded that the use of metal trays is superior regarding the dimensional accuracy and reliability of impression making, and should therefore be preferred. Lacy et al. ${ }^{18}$ also stated, accuracy and consistency were best maintained by the use of custom tray and adhesives to retain polyvinyl siloxanes. Corso et al. ${ }^{14}$ suggest the use of perforated trays, as the perforations can augment the effect of the adhesive and may improve accuracy.

All the impressions were poured after 1 hour of setting. According to Franco et al. ${ }^{11}$ delay in pouring will allow the material to recover elastically and release of by-products may influence the accuracy.

Present study showed an increase in interabutment distance with all impression materials tested in group I (putty-light body), group II (heavy body-light body) and group III (regular body-light body) compared with metal model. These findings indicate that there is increase in interabutment distance which can be attributed to firstly, the polymerization shrinkage of the material. Because of the constraint imposed by an effective adhesive on uniform shrinkage upon setting, abutment in resultant cast may tend to be a greater distance apart than they actually were on the master model or in the mouth. Secondly, due to linear setting expansion of the die material throughout the entire bulk of the stone block. ${ }^{7,9}$

Present study showed a significant increase in height with all impression materials tested in group I, group II and group III compared with master model. During polymerization reaction, impression material shrinks towards the center of mass. The use of tray adhesives, would redirect this shrinkage towards the impression tray wall. This was in agreement with Gordon et al. ${ }^{7}$ and Hung et al. ${ }^{8}$ study. Mean height of group II and group III varied significantly from each other, while in other groups, comparison was not significant. In contrast, in a study by Nissan et al., ${ }^{13}$ Johnson and Craig et al., ${ }^{16}$ Linke et al., ${ }^{17}$ there was decrease in the vertical height when compared with metal model.

Present study showed an increase in diameter significantly with all impression materials tested in group I, group II and group III compared with master model. The increase in the dimensions can be attributed to the polymerization shrinkage of the impression material towards the largest bulk in the center and also to the polymerization shrinkage towards the impression tray walls. These results agree with Idris et al. ${ }^{9}$ and Kumar et al. ${ }^{10}$

For all groups, larger dimensions were observed when compared with the metal master model. According to Petersen and Asmussen ${ }^{15}$ this can be due to swelling of material of low viscosity which was associated with a diffusion of unreacted compounds from the material of high viscosity into the material of low viscosity. Variability may have resulted from variation in the measurement technique or the real differences from the metal master model in the resulting dies, due to expansion of gypsum, distortion of the impression, or shrinkage of the impression material. ${ }^{19}$

According to Kumari and Nandeeshwar ${ }^{22}$ study, vinyl polysiloxane impression materials are available in all viscosities: light, medium, heavy and putty. Higher filler loading may increase the accuracy and higher the viscosity, less the constriction. Low viscosity material showed the greatest changes due to their lower filler content. The result of the present study which showed puttylight body combination as the most accurate dimensionally is in agreement with this.

Slight positive discrepancies, such as those observed in singlestep technique, are acceptable and may be advantageous in some situations, when larger dies may compensate the contraction developed during metal casting. ${ }^{20}$ 
In the present in vitro study, the inference can be derived as, the impression viscosity can be an important factor in determining the dimensional accuracy.

\section{Conclusion}

Within the limitations of this in vitro study, it is concluded that onestep putty-light body combination impression produced more accurate casts as compared to one-step heavy body-light body and regular body-light body combinations. The one-step technique has the advantages of simplicity and reasonable economy, however, in this technique, the putty tends to push the light body wash off the prepared tooth and thus critical areas, such as the finish line can be covered by the putty, which cannot record details to satisfactory levels.

\section{Limitations of the Study}

The dimensions with respect to the inter-abutment distance, the height of abutment and the diameter of abutment in each group (group I, II and III) were larger in dimensions as compared to metal master model (control group).

Only one brand of PVS material was used. The results may vary with different brands.

This study did not comment about the accuracy of the technique of impression making. The one-step technique has the advantages of simplicity and reasonable economy, however, in this technique, the tray material (putty, heavy body, regular body) tends to push the syringe material (light body) to wash off the prepared tooth and thus critical areas, such as the finish line can be covered by the tray material, which cannot record details to satisfactory.

\section{Clinical Significance}

Impression making to duplicate oral condition and tooth morphology is an integral part of prosthetic dentistry. Any failure in doing so, will hamper the treatment outcome. So it is very important to have knowledge about the dimensional accuracy of the impression material in use.

\section{References}

1. Walker MP, Ries D, Borello B. Implant cast accuracy as a function of impression techniques and impression materials viscosity. Int J Oral Maxillofac Implants 2008;23(4):669-674.

2. Singh K, Sahoo S, Prasad KD, et al. Effect of different impression techniques on the dimensional accuracy of impressions using various elastomeric impression materials: an in vitro study. J Contemp Dent Pract 2012;13(1):98-106.

3. Pandey A, Mehtra A. Comparative study of dimensional stability and accuracy of various elastomeric materials. IOSR Journal of Dental and Medical Sciences2014;13(3):40-44.
4. Mahalaxmi S. Materials used in dentistry. 1st ed., Wolters Kluwer (India) Pvt. Ltd; 2013.

5. Raigrodski AJ, Dogan S, Mancl LA, et al. A clinical comparison of two vinyl polysiloxane impression materials using the one-step technique. J Prosthet Dent 2009;102(3):179-186.

6. Rosenstiel S, Land M, Fujimoto J. Contemporary fixed prosthodontics. 4th ed., Mosby-Elsevier; 2011.

7. Gordon GE, Johnson GH, Drennon DG. The effect of tray selection on the accuracy of elastomeric impression materials. J Prosthet Dent 1990;63(1):12-15.

8. Hung SH, Purk JH, Tira DE, et al. Accuracy of one-step versus two-step putty wash addition silicone impression technique. J Prosthet Dent 1992;67(5):583-589.

9. Idris B, Houston F, Claffey N. Comparison of the dimensional accuracy of one and two-step techniques with the use of putty/wash addition silicone impression material. J Prosthet Dent 1995;74(5):535-541.

10. Kumar V, Aeran $\mathrm{H}$. Evaluation of effect of tray space on the accuracy of condensation silicone, addition silicone and polyether impression materials: an in vitro study. J Indian Prosthodont Soc 2012;12(3): 154-160.

11. Franco EB, da Cunha LF, Benetti AR. Effect of storage period on the accuracy of elastomeric impressions. J Appl Oral Sci 2007;15(3): 195-198.

12. Balkenhol M, Ferger P, Wöstmann B. Dimensional accuracy of 2-stage putty-wash impressions: influence of impression trays and viscosity. Int J Prosthodont 2007;20(6):573-575.

13. Nissan J, Laufer BZ, Brosh T, et al. Accuracy of three polyvinyl siloxane putty-wash impression techniques. J Prosthet Dent 2000;83(2): 161-165.

14. Corso M, Abanomy A, Canzio JD, et al. The effect of temperature changes on the dimensional stability of polyvinyl siloxane and polyether impression materials. J Prosthet Dent 1998;79:626-631.

15. Petersen GF, Asmussen E. Distortion of impression material used in the double mix techniques. Scand J Dent Res 1991;99:343-348.

16. Johnson GH, Craig RG. Accuracy of four types of rubber impression materials compared with time of pour and a repeat pour of models. J Prosthet Dent 1985;53(4):484-490.

17. Linke BA, Nicholls Ji, Faucher RR. Distortion analysis of the stone casts made from impression materials. J Prosthet Dent 1985;54(6): 794-802.

18. Lacy AM, Fukui H, Bellman T, et al. Time dependent accuracy of elastomeric impression materials. Part II: polyether, polysulfide and polyvinylsiloxane. J Prosthet Dent 1981;45(3):329-333.

19. Cayouette MJ, Burgess JO, Jones RE, et al. Three dimensional analysis of dual arch impression trays. Quintessence International 2003;34(3):189-198.

20. Franco EB, da Cunha LF, Herrera FS, et al. Accuracy of singlestep versus 2-step double-mix impression technique. ISRN Dent 2011;34:15-46.

21. Hassan AK. Dimensional accuracy of 3 silicone dental impression materials. East Mediterr Health J 2006;12:632-636

22. Kumari N, Nandeeshwar DB. The dimensional accuracy of polyvinyl siloxane impression materials using two different impression techniques: An in vitro study. J of Ind Prosthodontic Soc 2015;15(3):211-217. 